\title{
OPINION
}

\section{Time to future-proof plants in storage}

\author{
Seed banks must collect and condition their holdings of wild species so that they can thrive in landscapes \\ transformed by climate change, say Jeffrey Walck and Kingsley Dixon.
}

W hen the Millennium Seed Bank Partnership (MSBP) of the Royal

Botanic Gardens in Kew added its 24,200 th species to its collection this October - a Chinese pink banana - it completed one of the major tasks set at its inception in 2000: to store seeds from $10 \%$ of the world's known wild flora. Its next target is even more ambitious: to bank a quarter of all wild plant species by 2020 . Holding seeds against the immediate threat of extinction is crucial to preserving the world's plant diversity. Yet storage is only part of the challenge. Seed banks will have to do much more to re-establish species in landscapes that, because of global warming, will have changed irrevocably since they were collected.

The MSBP and other seed banks such as the Svalbard Global Seed Vault in the Arctic, nicknamed the 'doomsday vault', endeavour to collect samples that represent the normal genetic diversity of a species. At low temperatures, seeds can remain viable for hundreds if not thousands of years. Herein lies the problem: such seeds are literally frozen in time, a snapshot of the genetic diversity of a species at a particular point. Attempts to revive a seed in future habitats very different from those in which it developed could be doomed to failure. A germinating seed is genetically programmed to respond to a precise interplay of temperature and moisture that determines the climatic conditions in which it can best develop. In an environment that lacks the right temperature and moisture balance - which is likely to be disrupted by climate change - a seed will either fail to germinate or the seedling will perish soon afterwards (see Fig. 1).

Seed banks are expert at the preservation of wild seed. To prevent their samples becoming museum pieces they also need to take responsibility for the way the seeds are collected and for preparing them for planting back in the wild. Shrewd collection strategies can help to ensure that species are better adapted for replanting in habitats likely to differ substantially from their natural ranges. The primary aim should be to harvest as much genetic diversity as possible, so that collections are more likely to contain plants with traits suited to a changed climate.

One way to do this is to collect species at the limits of their ranges, where plants often

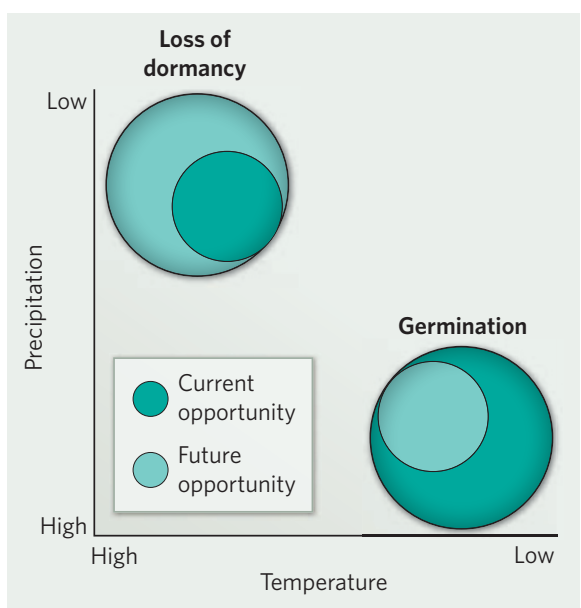

Figure 1 Changes in climate could affect the success of stored seeds. For seeds that need warm, dry conditions for dormancy to end, and cool, moist conditions to germinate - such as Western Australia's kangaroo paw - future warming and drying may enhance dormancy loss, but offer a smaller window for germination.

have higher levels of genetic novelty - for example, Mediterranean floras at the arid margins of their habitats, or mountain floras at the upper and lower limits of their altitude ranges. Another strategy is to collect samples from the same locations at different points in time, say every 10 or 20 years, because, as the climate changes, wild populations will produce natural variants that are better adapted to the new conditions.

\section{Mixing varieties}

Once gathered, much can be done to condition seeds for reintroduction to the wild. Most restoration nurseries propagate plants from stored seeds under glasshouse conditions without the robust selection processes of natural

\section{"Harvest as much} genetic diversity as possible." conditions, so it will be necessary to manipulate the growing environment and to screen for plants most suited to the new environment. There are established methods for doing this. For instance, subjecting seedlings to increased temperatures could allow the selection of those with higher heat tolerance. Likewise, subjecting seedlings to dry conditions could increase drought tolerance in subsequent generations. Both approaches could require multiple regeneration cycles at significant cost.

When the time comes to replant wild habitats, restoration scientists can use several techniques to maximize the chances that seeds will thrive in an altered landscape. For example, models are available that will predict how climate change will alter the home range of a species, so restorers can be sure to reintroduce seeds in places that will match their particular germination requirements. Where a plant population is heading for extinction and there are no stored samples suitable for repopulating, one option is to mix seeds from across the species' natural range - for example in a mountain habitat, mix seeds from higher altitudes with those from lower down - and plant them directly in the wild, allowing them to cross-breed naturally and spread traits that might help them survive in the new conditions.

To ensure that seeds are relevant to a climate-changed world, seed banks should team up with climate modellers to predict future vegetation patterns, and focus on keystone species, rare plants or those endemic to particular areas, that are particularly threatened by climate change. Policy-makers must get involved too: a comprehensive international agreement is required to coordinate the collection of genetic material, particularly for cross-border wild species. Finally, seed banks need to think big: rather than collecting 100 grams or so of each variety as is the common practice today, they should be collecting larger amounts, even up to 100 kilograms for some species such as palms with large seeds. This will mean that seed banks of the future will need to be considerably larger to accommodate bulk collections. Only in this way will they capture sufficient genetic diversity to rebuild the world's ecosystems.

Meeting the challenges of seed banking in the twenty-first century will be laborious and expensive, but there is no time to lose if we are to protect the planet's plant life from the rapid environmental change it now faces. Jeffrey Walck is in the Department of Biology, Middle Tennessee State University, Murfreesboro, Tennessee 37132, USA.

Kingsley Dixon is in the School of Plant Biology, the University of Western Australia, and director of science at Kings Park and Botanic Garden, West Perth 6005, Australia.

e-mails:jwalck@mtsu.edu; kdixon@bgpa.wa.gov.au 